\section{BLADDER CANCER}

\section{BLUE LIGHT IMPROVES DETECTION}

Blue light cystoscopy using hexaminolevulinate (HAL) improves detection of bladder cancer compared with white light cystoscopy in a real-world setting.

This study included 533 patients with suspected or known non-muscleinvasive bladder cancer undergoing transurethral resection of the bladder tumour (TURBT) and blue light and white light cystoscopy. Participants were enrolled onto a prospective registry. HAL was instilled into the bladders of participants $1-3 \mathrm{~h}$ before TURBT. Cystoscopic examination of the whole bladder was performed using white light and then repeated using blue light. Primary outcomes were sensitivity and specificity of blue light and white light cystoscopy alone and in combination.

In total, 1,632 pathology samples were obtained from 641 blue light cystoscopy procedures. Repeat procedures were performed in $16 \%$ of patients. Using final pathology as the reference standard, the sensitivity of white light cystoscopy was $76 \%$ and of blue light cystoscopy was $91 \%$; sensitivity of the combination was $99 \%$. Combining blue light cystoscopy with white light cystoscopy increased detection of papillary lesions by $12 \%$ and carcinoma in situ by $43 \%$.

An additional 206 lesions were detected in $25 \%$ of patients using blue light when the lesions were undectected using white light. Malignant lesions were detected exclusively using blue light in $8 \%$ of patients in whom white light found no tumours. Upward migration of American Urological Association risk group occurred in $6 \%$ of patients. The total rate of upgrading or upstaging resulting in a change in management using blue light cystoscopy was $14 \%$.

These results show that blue light cystoscopy for detection of bladder cancer is effective in a real-world setting.

Louise Stone

ORIGINAL ARTICLE Daneshmand, S. et al. Blue light cystoscopy for the diagnosis of bladder cancer: results from the US prospective multicenter registry. Urol. Onc. https://doi.org/ 10.1016/j.urolonc.2018.04.013 (2018)
PROSTATE CANCER

\section{[177Lu]PSMA-617 radionuclide therapy shows promise}

Several retrospective studies have reported the promising activity of radioconjugated prostate-specific membrane antigen (PSMA) antagonists in metastatic castration-resistant prostate cancer (mCRPC), but high-quality prospective data have been lacking. Now, a prospective phase II study reports that radionuclide treatment with [ $\left.{ }^{177} \mathrm{Lu}\right] \mathrm{PSMA}-617$ (LuPSMA) - a PSMA-targeted small molecule that emits DNA-damaging $\beta$-particles upon tumour cell internalization - has promising antitumour activity, low toxicity, and improves quality of life (QOL) in men with progressive $\mathrm{mCRPC}$.

The single-arm phase II trial recruited men with $\mathrm{mCRPC}$ who had progressive disease following taxane-based chemotherapy or second-generation antiandrogen therapy. All patients $(n=43)$ initially underwent PSMA imaging to confirm high PSMA expression at all metastatic sites, and eligible patients $(n=30)$ received up to four cycles of intravenous LuPSMA, according to tumour burden, with a mean administered radioactivity of 7.5 GBq per cycle. "We elected to adjust the amount of radioactivity based on tumour volume, renal function and body weight, adapted from our protocols using ${ }^{177} \mathrm{Lu}$ to treat neuroendocrine tumours," explains lead investigator Michael Hofman. This innovative 'theranostics' approach resulted in a highly personalized treatment strategy.

At a median follow up period of 25 months, the main primary end point of PSA response (defined as $\geq 50 \%$ PSA decline) was achieved in $17(57 \%)$ of 30 patients $(95 \% \mathrm{Cl} 37-75)$. Of the 17 patients with measurable nodal or visceral disease on imaging at baseline, 14 (82\%) had an objective response, 5 (29\%) had a complete response, and 9 (53\%) had a partial response. PSA progression occurred in 27 (90\%) patients, and median biochemical progression-free survival (bPFS) and overall survival (OS) were 7.6 months $(95 \% \mathrm{Cl} 6.3-9.0)$ and 13.5 months $(95 \% \mathrm{Cl}$ 10.4-22.7), respectively. Interestingly, a post-hoc analysis revealed that $\mathrm{a} \geq 50 \%$ PSA decline was associated with even greater improvements in bPFS and OS.

"For a palliative therapy, the treatment was well tolerated with improvements in patient-reported QOL measures observed," says Hofman. Indeed, in the 27 (90\%) patients with pain at baseline, mean pain scores improved in both severity and interference at all time points.

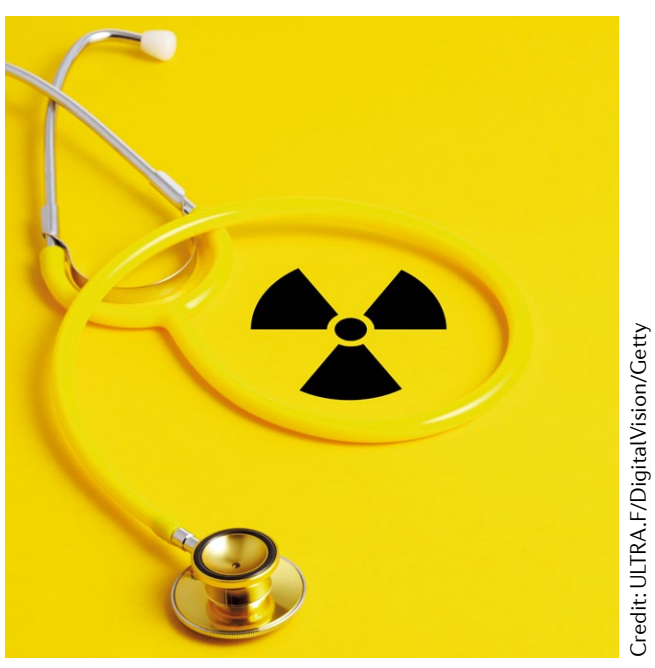

Moreover, 11 (37\%) patients experienced a $\geq 10$-point improvement in global health score by the second LuPSMA cycle. Regarding safety and toxicity, LuPSMA was well tolerated with no treatment-related deaths. The most common treatment-related adverse events were grade 1 xerostomia in $26(87 \%)$ patients, grade 1-2 transient nausea in $15(50 \%)$ patients, and grade $1-2$ fatigue in $15(50 \%)$ patients. Only four (13\%) patients reported grade 3-4 thrombocytopenia, which was possibly due to LuPSMA treatment.

Overall, the findings offer compelling evidence for the safety and efficacy of LuPSMA in progressive, PSMA-high mCRPC, and are particularly promising given the almost ubiquitous expression of PSMA in mCRPC. On the basis of these data, the multicentre, randomized phase II ANZUP/PCFA TheraP Trial (NCT03392428) has commenced to compare LuPSMA versus cabazitaxel in men with $\mathrm{mCRPC}$ who progressed after docetaxel. "Endocyte, who purchased PSMA-617 last year, will also shortly commence a multicentre phase III registration trial," adds Hofman. "We are also interested in combining LuPSMA with other treatments to further increase efficacy, and are planning two trials combining LuPSMA with poly(ADP-ribose) polymerase (PARP) inhibitors and anti-programmed cell death protein 1 (PD1) therapy."

Conor A. Bradley

ORIGINAL ARTICLE Hofman, M. S. et al. [177Lu]-PSMA-617 radionuclide treatment in patients with metastatic castrationresistant prostate cancer (LuPSMA trial): a single-centre, single-arm, phase 2 study. Lancet Oncol. https://doi.org/10.1016/S14702045(18)30198-0 (2018) 